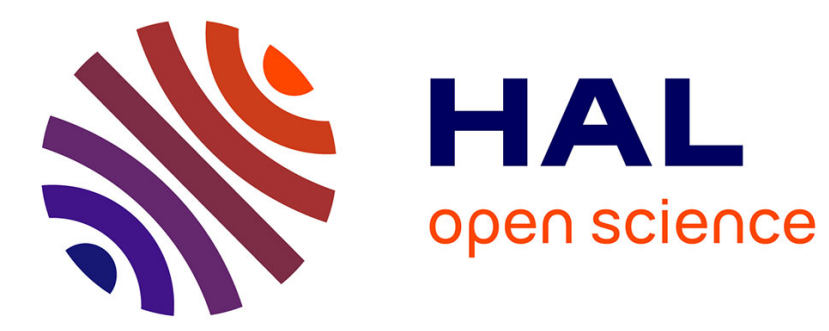

\title{
Ressources renouvelables et incertitude sur lespréférences des générations futures
}

\author{
Alain Ayong Le Kama, Katheline Schubert
}

\section{To cite this version:}

Alain Ayong Le Kama, Katheline Schubert. Ressources renouvelables et incertitude sur lespréférences des générations futures. Revue d'Economie Politique, 2006, 116 (2), pp.229-250. halshs-00206513

\section{HAL Id: halshs-00206513 \\ https://shs.hal.science/halshs-00206513}

Submitted on 17 Jan 2008

HAL is a multi-disciplinary open access archive for the deposit and dissemination of scientific research documents, whether they are published or not. The documents may come from teaching and research institutions in France or abroad, or from public or private research centers.
L'archive ouverte pluridisciplinaire HAL, est destinée au dépôt et à la diffusion de documents scientifiques de niveau recherche, publiés ou non, émanant des établissements d'enseignement et de recherche français ou étrangers, des laboratoires publics ou privés. 


\title{
Ressources renouvelables et incertitude sur les préférences des générations futures
}

\author{
Alain Ayong Le Kama* $\quad$ Katheline Schubert ${ }^{\dagger}$
}

Septembre 2005

\begin{abstract}
Résumé
Rien ne nous permet d'être certains que les générations futures auront les mêmes préférences que nous concernant les biens environnementaux. En outre, même si nous sommes tentés, en raison de la dégradation de la qualité de l'environnement, de croire que leur sensibilité à l'environnement sera plus grande que la notre, rien ne nous permet de l'affirmer avec certitude. Cet article étudie alors, dans un cadre très simple, les conséquences en termes de trajectoires optimales de consommation d'une incertitude sur les préférences des générations futures en matière de consommation d'une ressource environnementale renouvelable. Une telle incertitude va-telle induire un comportement optimal plus "conservateur" vis-à-vis de la ressource? Nous montrons que tel est le cas uniquement si nous anticipons aujourd'hui que les générations futures seront en moyenne plus sensibles que nous ne le sommes à la consommation permise par l'utilisation de la ressource environnementale. Nous montrons en outre que la prise en compte de cette incertitude conduit à une profonde modification de la trajectoire optimale de consommation, qui non seulement devient non-continue mais en outre peut devenir non-monotone.
\end{abstract}

Mots-clés : ressources renouvelables, croissance, préférences, incertitude

Classification JEL : O41, Q20

*Université de Lille 1 et Commissariat général du Plan. e-mail : adayong@univ-paris1.fr

${ }^{\dagger}$ EUREQua, Université de Paris 1. e-mail : schubert@univ-paris1.fr

Adresse : EUREQua, Université de Paris 1, Maison des Sciences Economiques, 106-112 bd de l'Hôpital 75647 Paris cedex 13.

Nous tenons à remercier A. d'Autume et les deux rapporteurs de la revue pour leurs éclairantes remarques et suggestions. Nous restons néanmoins seuls responsables des éventuelles erreurs ou omissions. 


\title{
Renewable resources and uncertainty about future preferences
}

\begin{abstract}
The attitude of future generations towards environmental assets may well be different from ours. Even if we are tempted to believe that their sensitivity to environmental matters will be greater than ours, because of environmental depletion, we cannot make sure of that. This paper analyses the consequences of an uncertainty about future preferences in terms of the optimal consumption of a renewable resource in a very simple set-up. Will uncertainty lead to a more conservatice attitude towards the resource? We show that this is the case only when we expect now that future generations will be on average more sensitive than we are to the consumption of the resource. We also show that when we take this uncertainty into account the optimal path of consumption is deeply modified : not only does it become non-continuous but also it can become non-monotonous.
\end{abstract}

Key words : renewable resources, growth, preferences, uncertainty

JEL classification : O41, Q20 


\section{Introduction}

La difficulté inhérente à la prise de décisions en matière de politique environnementale aujourd'hui est, au moins pour partie, due à la grande ignorance dans laquelle nous sommes des conséquences à moyen et long terme des atteintes courantes à l'environnement (épuisement des ressources naturelles, accumulation des déchets, pollution de l'air et de l'eau, pertes de biodiversité...). A cette incertitude sur les conséquences s'ajoute une incertitude sur la perception de ces conséquences par les générations futures : il est fort possible que ces dernières aient des évaluations (ou des préférences) concernant les biens environnementaux différentes des notres. Il faut dès lors intégrer explicitement cette possibilité de modification de la sensibilité des générations futures à nos prises de décisions contemporaines.

L'introduction d'une incertitude sur les préférences des générations futures est assez récente dans la littérature sur l'économie de l'environnement. Si elle avait déjà sommairement été évoquée par Dasgupta et Heal (1974), elle n'a fait l'objet d'une étude spécifique que depuis le début des années quatre-vingt-dix. L'article fondateur est celui de Beltratti, Chichilnisky et Heal (1993), partiellement repris par Beltratti (1996) et approfondi par Beltratti, Chichilnisky et Heal (1998) et Ayong Le Kama (2001).

Ces travaux présentent un modèle de consommation optimale d'une ressource non renouvelable dans lequel le planificateur est confronté à deux types d'incertitudes. Il existe non seulement une incertitude exogène sur les préférences des générations futures, mais également une incertitude sur la date à laquelle se manifestera un changement dans les préférences. Ils comparent alors les trajectoires optimales de consommation de ce modèle avec celles qui prévalent lorsque les préférences ne changent pas au cours du temps. Ils montrent que la prise en compte de cette incertitude, sous réserve qu'elle soit asymétrique - c'est-à-dire que le planificateur anticipe qu'en moyenne les préférences des générations futures seront différentes de celles des générations courantes, peut conduire le planificateur à adopter, dès aujourd'hui, une attitude plus conservatrice ${ }^{1}$.

Nous étendons ici cette analyse au cas où la ressource possède un processus de régénération naturelle. Il est alors possible de voir cette ressource comme une ressource biologique renouvelable indispensable à la consommation, ou comme une qualité de l'environnement que l'activité humaine dégrade mais qui a la capacité de partiellement se régénérer de façon naturelle. Nos résultats sont alors à la fois plus généraux, grâce à l'introduction du caractère renouvelable de la ressource, et plus précis, en raison de la résolution analytique complète du modèle, que ceux des travaux cités précédemment ${ }^{2}$.

Afin de rendre plus transparent le problème posé et les conclusions qu'il est possible d'en inférer, nous décomposons l'analyse du problème.

Nous présentons tout d'abord le profil optimal de consommation dans le modèle qui nous servira de référence, c'est-à-dire le problème déterministe sans modification des pré-

\footnotetext{
${ }^{1}$ Nous considérerons que le planificateur adopte une attitude plus conservatrice qu'une attitude de référence si le rapport de la consommation au stock de ressource est, à chaque instant, plus faible le long de la trajectoire optimale que l'on étudie que le long de la trajectoire de référence. Ceci suppose, pour un stock de ressource initial donné, une consommation initiale plus faible que la consommation de référence.

${ }^{2}$ Ayong Le Kama et Schubert (2004) envisagent le cas plus général d'une fonction d'utilité non séparable dépendant de la consommation et de la qualité de l'environnement.
} 
férences.

Nous décrivons ensuite la trajectoire optimale de l'économie quand les préférences futures sont incertaines mais que la date d'apparition de l'éventuelle modification de préférences est donnée. Nous montrons dans ce cas que si l'incertitude est asymétrique ou, en d'autres termes, si le planificateur anticipe qu'en moyenne les préférences se modifieront dans le futur, il est plus conservateur avant le changement de préférences s'il pense que les générations futures seront plus sensibles à l'environnement que nous ne le sommes, et moins conservateur dans le cas contraire; en outre, au moment du changement de préférences apparaît un "saut" du niveau de consommation.

Nous analysons ensuite le problème complet, qui prend en compte les deux formes d'incertitudes ${ }^{3}$. Nous montrons que si l'incertitude est symétrique, le problème est équivalent au problème de référence et l'incertitude n'a aucun effet sur le comportement des agents. En revanche, si l'incertitude est asymétrique, elle affecte fortement les décisions du planificateur. Nous montrons alors que le profil de consommation non seulement devient non-continu - c'est-à-dire que la consommation "saute" comme dans le cas précédent mais aussi et surtout peut devenir non-monotone. Selon l'anticipation que fait le planificateur sur les préférences des générations à venir et en fonction de son impatience et de la capacité de la ressource naturelle à se renouveler, il peut adopter un profil de consommation croissant avant l'apparition du changement de préférences et décroissant après (ou l'inverse). Ceci tout en étant toujours plus (resp. moins) conservateur qu'en l'absence d'incertitude s'il anticipe que les générations futures valoriseront plus (resp. moins) la ressource naturelle que les générations présentes.

\section{Le modèle}

Nous considérons une économie d'échange dans laquelle un agent représentatif à durée de vie infinie consomme une unique ressource environnementale ${ }^{4}$. La dynamique d'accumulation du stock de la ressource environnementale est la suivante :

$$
\dot{R}_{t}=\delta R_{t}-C_{t}
$$

où $R_{t} \geq 0$ et $C_{t} \geq 0$ sont respectivement le stock de ressource et le flux consommé à l'instant $t$. Cette ressource a une capacité de régénération naturelle $\delta>0$ que l'on suppose, pour simplifier et malgré le caractère réducteur de cette hypothèse, certaine et constante.

Les préférences courantes de l'agent sont caractérisées par une fonction d'utilité instantanée, $U(C)$, qui ne dépend que du niveau de consommation courante, avec $U($.) croissante, deux fois continûment différentiable et strictement concave, et $U^{\prime}(0)=+\infty$.

Nous supposons, comme l'ont fait Beltratti, Chichilnisky et Heal (1993), Beltratti (1996) ou encore Beltratti, Chichilnisky et Heal (1998), que l'économie est confrontée à une double dimension d'incertitude.

\footnotetext{
${ }^{3}$ Le problème précédent ne peut pas nécessairement être considéré comme un cas particulier de ce problème général, ce qui justifie son étude.

${ }^{4}$ Classiquement, cet agent à durée de vie infinie symbolise la succession d'une infinité de générations d'agents identiques parfaitement altruistes.
} 
(i) Il est possible que dans le futur l'utilité procurée par la consommation du bien environnemental se modifie dans un sens inconnu.

Dit autrement, à partir d'une date $T$ donnée, il est possible que l'évaluation par les générations futures du bien environnemental vienne à changer. Celles-ci peuvent ainsi devenir plus sensibles à la consommation permise par la ressource environnementale que les générations présentes. Dans ce cas, qui se produira avec une probabilité exogène $q$, leur utilité passera pour tout niveau de consommation $C$ de $U(C)$ à $(1+\alpha) U(C), \alpha>$ 0 . Elles peuvent également, avec une probabilité $(1-q)$, devenir moins sensibles à la consommation de ressource environnementale, leur utilité passant de $U(C)$ à $(1-\beta) U(C)$, $\beta>0$. Nous imposons $\beta<1$ afin d'assurer que la consommation permise par la ressource environnementale soit source de satisfaction et non de désutilité.

(ii) La date $T$ à laquelle survient le changement de préférences est elle-même aléatoire.

Nous notons $f_{t}$ la fonction de densité de probabilité de la variable aléatoire $T$, avec $f_{t}>0$ (la probabilité pour que les préférences se modifient en $t$ est positive pour tout $t$ ) et $\int_{0}^{\infty} f_{t} d t=1$ (le changement de préférences survient de manière certaine à une date finie). On note $F_{t}=\int_{0}^{t} f_{s} d s$ la fonction de répartition associée à $f$. En conséquence, $1-F_{t}=\int_{t}^{\infty} f_{s} d s$ représente la probabilité qu'à la date $t$ le changement de préférences ne soit pas encore advenu.

Afin de poser clairement le problème que doit résoudre le planificateur, il nous faut commencer par décrire le problème auxiliaire, proposé par Dasgupta et Heal (1974), suivant :

$$
\left\{\begin{array}{l}
V_{\Lambda}\left(R_{T}\right)=\max \int_{T}^{\infty} \Lambda\left(C_{t}\right) e^{-\rho(t-T)} d t \\
\text { sc. } \mid \begin{array}{l}
\dot{R}_{t}=\delta R_{t}-C_{t} ; t \geq T \\
R_{t} \geq 0 ; R_{T}(>0) \text { donné }
\end{array}
\end{array}\right.
$$

avec $\rho>0$ le taux d'escompte appliqué aux utilités futures ${ }^{5}$. Dans la formulation (2), $V_{\Lambda}\left(R_{T}\right)$ représente la fonction valeur du problème et se ramène à la valeur du stock de ressource subsistant à la date $T$ du changement de préférences. Enfin, $\Lambda($.) représente la fonction d'utilité qui prévaudra à la suite du changement dans les préférences.

On note $V($.$) la fonction V_{\Lambda}($.$) quand \Lambda()=.U($.$) . Si le changement de préférences est$ favorable à la ressource environnementale, $\Lambda()=.(1+\alpha) U($.$) et la fonction valeur devient$ $V_{\Lambda}\left(R_{T}\right)=(1+\alpha) V\left(R_{T}\right)$. Dans le cas opposé, la fonction d'utilité après le changement de préférences devient $\Lambda()=.(1-\beta) U($.$) et la fonction valeur V_{\Lambda}\left(R_{T}\right)=(1-\beta) V\left(R_{T}\right)$.

En notant $E$ l'opérateur d'espérance mathématique, on peut définir la valeur espérée du stock de ressource à la date $T$ comme suit :

$$
E V_{\Lambda}\left(R_{T}\right)=q(1+\alpha) V\left(R_{T}\right)+(1-q)(1-\beta) V\left(R_{T}\right)=\Gamma V\left(R_{T}\right)
$$

où le paramètre $\Gamma$ est défini par $\Gamma=q(1+\alpha)+(1-q)(1-\beta)>0$.

L'objectif du planificateur tient en la maximisation de l'espérance mathématique de

\footnotetext{
${ }^{5}$ Pour une discussion sur l'opportunité d'escompter les préférences futures dans ce contexte, $c f$. Heal (1985).
} 
la somme actualisée des utilités, et son programme s'écrit donc :

$$
\mathcal{P}\left\{\begin{array}{l|l}
\max \int_{0}^{\infty} f_{T}\left[\int_{0}^{T} U\left(C_{t}\right) e^{-\rho t} d t+e^{-\rho T} \Gamma V\left(R_{T}\right)\right] d T \\
\text { sc. } \mid \begin{array}{l}
\dot{R}_{t}=\delta R_{t}-C_{t} \\
R_{0} \text { donné } ; R_{t} \geq 0, C_{t} \geq 0
\end{array}
\end{array}\right.
$$

\subsection{La consommation optimale en l'absence d'incertitude}

Nous considérons dans un premier temps le cas où il n'y a ni incertitude ni changement de préférences. Nous retrouvons alors le problème déterministe standard suivant :

$$
\mathcal{P}[1] \quad\left\{\begin{array}{l}
V\left(R_{0}\right)=\max \int_{0}^{\infty} U\left(C_{t}\right) e^{-\rho t} d t \\
\text { sc. } \mid \begin{array}{l}
\dot{R}_{t}=\delta R_{t}-C_{t} \\
R_{0} \text { donné } ; R_{t} \geq 0, C_{t} \geq 0
\end{array}
\end{array}\right.
$$

On note $\sigma=-\frac{U^{\prime}(C)}{C U^{\prime \prime}(C)}>0$ l'élasticité de substitution intertemporelle de la consommation et on suppose cette élasticité constante, de façon à obtenir des sentiers de croissance à taux constant. On note en outre $\lambda_{t}$ le prix implicite de la ressource environnementale à l'instant $t$. Il est alors aisé de montrer que la solution du problème déterministe $\mathcal{P}$ [1] est caractérisée par les relations suivantes :

$$
\begin{aligned}
& \frac{\dot{C}_{t}^{[1]}}{C_{t}^{[1]}}=g^{[1]}=g=\sigma(\delta-\rho) \\
& C_{t}^{[1]}=C_{0}^{[1]} e^{g t}=(\delta-g) R_{0} e^{g t} \\
& R_{t}^{[1]}=R_{0} e^{g t} \\
& \lambda_{t}^{[1]}=\lambda_{0} e^{(\rho-\delta) t}=U^{\prime}\left(C_{t}^{[1]}\right)
\end{aligned}
$$

La stricte positivité de la consommation exige $\delta-g>0$, soit $(1-\sigma) \delta+\sigma \rho>0$. Si l'élasticité de substitution intertemporelle de la consommation est inférieure à l'unité, cette condition est toujours vérifiée. En revanche, si elle est supérieure à l'unité, le taux d'escompte social doit être suffisamment élevé par rapport au taux de régénération de la ressource pour que cette condition soit vérifiée. En outre, on peut montrer que c'est cette même condition qui assure le respect de la condition de transversalité. Nous la supposons donc vérifiée. Alors, les conditions nécessaires d'optimalité sont aussi suffisantes.

Les niveaux de consommation et de ressource évoluent au même taux constant $g$, qui peut être positif si la capacité de régération naturelle de la ressource est suffisamment grande par rapport à l'impatience des agents, et qui est négatif dans le cas contraire. Le rapport de la consommation au stock de ressource est constant et vaut $\delta-g=(1-\sigma) \delta+\sigma \rho$. Il est d'autant plus élevé que la capacité de régération naturelle et l'impatience des agents sont grandes. 


\subsection{La consommation optimale quand la date de changement de préférences est certaine}

Nous considérons maintenant qu'il existe une incertitude sur les préférences futures mais que la date à laquelle apparaîtra le changement est connue par le planificateur. Le problème $\mathcal{P}$ donné en (4) devient dans ce cas :

$$
\mathcal{P}[3] \quad\left\{\begin{array}{l}
\max \int_{0}^{T} U\left(C_{t}\right) e^{-\rho t} d t+e^{-\rho T} \Gamma V\left(R_{T}\right) \\
\text { sc. } \mid \begin{array}{l}
\dot{R}_{t}=\delta R_{t}-C_{t} \\
R_{0} \text { donné } ; R_{t} \geq 0, C_{t} \geq 0
\end{array}
\end{array}\right.
$$

En raison de la séparabilité du problème $\mathcal{P}[3]$, due à la forme additive de la fonction objectif, le profil de consommation global est divisé en deux parties. Avant la date $T$ du changement de préférences, la consommation optimale est issue de la résolution du problème $\mathcal{P}[3]$. Après cette date, elle est donnée par le programme $\mathcal{P}[2]$ suivant :

$$
\mathcal{P}[2] \quad\left\{\begin{array}{l}
V\left(R_{T}\right)=\max \int_{T}^{\infty} U\left(C_{t}\right) e^{-\rho(t-T)} d t \\
\text { sc. } \mid \begin{array}{l}
R_{t}=\delta R_{t}-C_{t} \quad ; t \geq T \\
R_{T} \text { donné } ; R_{t} \geq 0, C_{t} \geq 0
\end{array}
\end{array}\right.
$$

En effet, la trajectoire optimale pour ce problème est la même que la trajectoire optimale pour $\Gamma V\left(R_{T}\right), \Gamma$ étant strictement positif.

\section{La trajectoire optimale de consommation après la date de changement de préférences}

Par analogie avec $\mathcal{P}[1]$, la solution de $\mathcal{P}[2]$ est donnée par les relations suivantes :

$$
\begin{aligned}
& \frac{\dot{C}_{t}^{[2]}}{C_{t}^{[2]}}=g^{[2]}=g^{[1]}=g \\
& C_{t}^{[2]}=C_{T}^{[2]} e^{g(t-T)}=(\delta-g) R_{T} e^{g(t-T)} \\
& R_{t}^{[2]}=R_{T} e^{g(t-T)} \\
& \lambda_{t}^{[2]}=\lambda_{T}^{[2]} e^{(\rho-\delta)(t-T)}=U^{\prime}\left(C_{t}^{[2]}\right)
\end{aligned}
$$

Le taux de croissance commun de la consommation et du stock de ressource est le même qu'en l'absence de changement de préférences. La raison en est que le paramètre de préférence qui joue sur le taux de croissance de la consommation est l'élasticité de substitution intertemporelle de la consommation ; or celle-ci n'est pas, sous nos hypothèses, affectée par le changement de préférences.

Nous pouvons par ailleurs montrer qu'au moment du changement de préférences, la valeur marginale de la ressource est égale à son prix implicite ( $c f$. annexe 1), soit :

$$
V^{\prime}\left(R_{T}\right)=\lambda_{T}^{[2]}
$$

Il est important de noter que le sentier de consommation [2] est à la fois celui que le planificateur prévoit à l'instant 0 de faire suivre à l'économie à partir de l'instant $T$, sans 
connaître le sens du changement de préférences, et celui que l'économie suivra effectivement à partir de l'instant $T$, une fois l'incertitude résolue. En effet, après résolution de l'incertitude, l'économie dispose d'un stock de ressource $R_{T}$ connu. Que les préférences soient $(1+\alpha) U(C)$ ou $(1-\beta) U(C)$, le stock de ressource et donc la consommation évolueront, à partir du stock $R_{T}$, au même taux $g$. La présence d'incertitude n'influe donc que sur le "point de départ" du sentier de consommation postérieur au changement de préférences, ou, dit autrement, que sur le legs que les générations présentes vont consentir aux générations futures.

\section{La trajectoire optimale de consommation avant la date de changement de pré- férences}

La méthode de résolution est la même. Il faut cependant ajouter aux conditions nécessaires d'optimalité la condition de transversalité qui peut se reformuler, en utilisant la relation (16), comme suit :

$$
\lambda_{T}^{[3]}=\Gamma V^{\prime}\left(R_{T}\right)=\Gamma \lambda_{T}^{[2]}
$$

Ainsi, au moment du changement de préférences, un saut va se produire dans la trajectoire du prix implicite de la ressource environnementale.

Nous savons par ailleurs, d'après les conditions d'optimalité, que pour chacune des composantes de la trajectoire de consommation, soit [3] avant le changement, et [2] après le changement, on a égalité entre l'utilité marginale de la consommation et le prix implicite de la ressource à chaque date $t$. La condition de transversalité se résume alors à :

$$
U^{\prime}\left(C_{T}^{[3]}\right)=\Gamma U^{\prime}\left(C_{T}^{[2]}\right)
$$

Nous avons supposé l'élasticité de substitution intertemporelle de la consommation constante. La fonction d'utilité est donc CRRA $\left(U(C)=\frac{C^{1-\frac{1}{\sigma}}}{1-\frac{1}{\sigma}}\right)$ et la condition de transversalité (18) permet d'obtenir la relation suivante entre les niveaux de consommation au moment du changement de préférences :

$$
C_{T}^{[3]}=\Gamma^{-\sigma} C_{T}^{[2]}
$$

Nous montrons en annexe 2 que la trajectoire optimale de consommation est alors donnée par :

$$
C_{t}^{[3]}=(\delta-g) e^{g(t-T)} \Gamma^{-\sigma} R_{T}
$$

avec :

$$
R_{T}=\frac{R_{0}}{e^{-g T} \Gamma^{-\sigma}+e^{-\delta T}\left(1-\Gamma^{-\sigma}\right)}
$$

L'annexe 2 montre également que le stock de ressource évolue de la façon suivante :

$$
R_{t}^{[3]}=R_{0} e^{g t} \frac{1+e^{(\delta-g)(t-T)}\left(\Gamma^{\sigma}-1\right)}{1+e^{-(\delta-g) T}\left(\Gamma^{\sigma}-1\right)}
$$

Il ne croît donc plus à taux constant le long de la trajectoire antérieure au changement de préférences.

Nous commentons plus loin ces résultats. Notons simplement que le legs environnemental consenti par les générations présentes aux générations futures $R_{T}$ est d'autant plus élevé que $\Gamma$ est élevé, conformément à l'intuition. 


\subsection{La résolution du modèle complet}

L'objectif du planificateur dans le modèle complet, décrit par le problème $\mathcal{P}$ (équation (4)), revient à maximiser l'espérance mathématique de l'utilité actualisée de la consommation de la ressource environnementale. L'espérance est prise par rapport aux distributions de probabilité gouvernant les changements de préférences et la date d'apparition de ces changements. Notons tout d'abord que quand l'incertitude est symétrique il est aisé de se ramener au problème déterministe : une incertitude symétrique n'a aucun effet sur la trajectoire sélectionnée par le planificateur. Nous étudions donc dans ce qui suit le cas d'une incertitude asymétrique.

Par une intégration par partie, on montre (cf. Dasgupta et Heal (1974)) que l'objectif à maximiser du problème $\mathcal{P}$ se ramène à $\int_{0}^{\infty} e^{-\rho t}\left[\left(1-F_{t}\right) U\left(C_{t}\right)+f(t) \Gamma V\left(R_{t}\right)\right] d t$. Le problème à résoudre devient alors :

$$
\mathcal{P}[4] \quad\left\{\begin{array}{l}
\max \int_{0}^{\infty} e^{-\rho t}\left[\left(1-F_{t}\right) U\left(C_{t}\right)+f_{t} \Gamma V\left(R_{t}\right)\right] d t \\
\text { sc. } \mid \begin{array}{l}
\dot{R}_{t}=\delta R_{t}-C_{t} \\
R_{0} \text { donné } ; R_{t} \geq 0, C_{t} \geq 0
\end{array}
\end{array}\right.
$$

Comme dans le cas d'une date de changement certaine, les choix optimaux s'effectuent en deux étapes. La date de changement de préférences étant cependant aléatoire, la valeur du legs, $R_{t}$, en devient incertaine. Une fois que s'est manifesté le changement en $t$, les trajectoires optimales de consommation sont obtenues par la résolution du problème déterministe $\mathcal{P}[2]$.

Si l'on note $\mu_{t}=\frac{f_{t}}{1-F_{t}}$ la probabilité conditionnelle que le changement se produise en $t$ sachant qu'il ne s'est pas manifesté plus tôt, on montre en annexe 3 que le profil de consommation optimale est donné par :

$$
\frac{\dot{C}_{t}^{[4]}}{C_{t}^{[4]}}=g-\sigma \mu_{t}\left[1-\frac{\Gamma V^{\prime}\left(R_{t}\right)}{U^{\prime}\left(C_{t}^{[4]}\right)}\right]
$$

Le taux de croissance de la consommation, noté $g^{[4]}$, n'est ainsi plus constant; il dépend non seulement de la probabilité que le changement de préférences survienne en $t$, mais aussi du rapport entre l'espérance de la valeur marginale de la ressource, $\Gamma V^{\prime}\left(R_{t}\right)$, et l'utilité marginale de la ressource au moment du changement. Il se reformule selon :

$$
g_{t}^{[4]}=g+\tau_{t}
$$

$\tau_{t}=-\sigma \mu_{t}\left[1-\frac{\Gamma V^{\prime}\left(R_{t}\right)}{U^{\prime}\left(C_{t}^{[4]}\right)}\right]$ représentant la partie du taux de croissance qui dépend du temps et synthétisant la composante incertaine de ce taux.

Pour mieux comprendre cette expression du taux de croissance de la consommation, il faut garder à l'esprit que la date de changement de préférences est maintenant la date $t$. Nous pouvons alors vérifier qu'à la date $t$, la valeur marginale de la ressource est, comme dans l'équation (16), égale à son prix implicite. La variable $\tau_{t}$ peut alors se réécrire comme suit :

$$
\tau_{t}=-\sigma \mu_{t}\left[1-\frac{\Gamma U^{\prime}\left(C_{t}^{[2]}\right)}{U^{\prime}\left(C_{t}^{[4]}\right)}\right]
$$


La valeur du taux de croissance de la consommation $g^{[4]}$ va par conséquent dépendre, pour une valeur donnée de $\Gamma$, du rapport entre les utilités marginales des consommations antérieures au changement (trajectoire [4]) et postérieures au changement (trajectoire [2]).

Nous supposons par ailleurs, pour simplifier, que la fonction $f_{t}$ est la densité de probabilité d'un processus de Poisson de paramètre $\mu>0$, ce qui implique que $\mu_{t}=\frac{f_{t}}{1-F_{t}}$, la probabilité conditionnelle que le changement se réalise en $t$ sachant qu'il ne s'est pas réalisé avant, est constante et égale à $\mu$. La variable $\tau_{t}$ ne dépend alors plus que du seul rapport des utilités marginales.

Nous savons (équations (13) et (14)) que l'on a, le long de la trajectoire postérieure au changement de préférences,

$$
C_{t}^{[2]}=(\delta-g) R_{t}
$$

où $R_{t}$ est le stock de ressource restant à l'instant $t$. En utilisant la forme CRRA de la fonction d'utilité, nous pouvons alors écrire l'équation (24) sous la forme suivante :

$$
\frac{\dot{C}_{t}^{[4]}}{C_{t}^{[4]}}=g-\sigma \mu\left[1-\frac{\Gamma(\delta-g)^{-\frac{1}{\sigma}} R_{t}^{-\frac{1}{\sigma}}}{C_{t}^{[4]-\frac{1}{\sigma}}}\right]
$$

Notons par ailleurs que l'équation d'accumulation du stock de ressource permet d'écrire, le long de la trajectoire antérieure au changement de préférences :

$$
\frac{\dot{R}_{t}}{R_{t}}=\delta-\frac{C_{t}^{[4]}}{R_{t}}
$$

Introduisons alors la variable $x_{t}=\frac{C_{t}^{[4]}}{R_{t}}$. Les deux équations précédentes nous permettent de déterminer aisément le taux de croissance de cette variable :

$$
\frac{\dot{x}_{t}}{x_{t}}=g-\sigma \mu\left[1-\Gamma(\delta-g)^{-\frac{1}{\sigma}} x_{t}^{\frac{1}{\sigma}}\right]-\delta+x_{t}
$$

Nous montrons en annexe 4 que cette équation admet un état stationnaire unique noté $x^{*}$ et que, cette équation étant instable, le ratio $C / R$ se place immédiatement à sa valeur stationnaire $x^{*}$. Le taux de croissance commun de la consommation et du stock de ressource vaut donc :

$$
g^{[4]}=\delta-x^{*}\left\{\begin{array}{l}
=g \text { si } \Gamma=1 \\
>g \text { si } \Gamma>1 \\
<g \text { si } \Gamma<1
\end{array}\right.
$$

et la valeur de la consommation initiale dans ce cas est simplement :

$$
C_{0}^{[4]}=x^{*} R_{0}=\left(\delta-g^{[4]}\right) R_{0}
$$




\subsection{La comparaison des trajectoires}

La comparaison des trajectoires issues des problèmes $\mathcal{P}[3]$, avec incertitude sur le sens du changement de préférences à une date donnée, et $\mathcal{P}$ [4], où l'on intègre les deux formes d'incertitude, à la situation de référence $\mathcal{P}[1]$ est rendue possible par le fait que toutes satisfont la même contrainte de ressource intertemporelle. L'ensemble des valeurs nécessaires à la réalisation de cette comparaison est résumé dans le tableau 1.

\section{TAB. 1 - LA COMPARAISON DES TRAJECTOIRES}

\begin{tabular}{|l|l|l|l|}
\hline & référence & cas $T \operatorname{certain}^{(b)}$ & modèle complet $^{(c)}$ \\
\hline$C_{0}$ & $C_{0}^{[1]}=(\delta-g) R_{0}$ & $C_{0}^{[3]}=\frac{C_{0}^{[1]}}{1+e^{(g-\delta) T}\left(\Gamma^{\sigma}-1\right)}$ & $C_{0}^{[4]}=x^{*} R_{0}=\frac{x^{*}}{\delta-g} C_{0}^{[1]}$ \\
\hline$C_{T}(-)^{(a)}$ & $C_{T}^{[1]}=(\delta-g) R_{0} e^{g T}$ & $C_{T}^{[3]}=\frac{C_{T}^{[1]}}{1+e^{(g-\delta) T}\left(\Gamma^{\sigma}-1\right)}$ & $C_{T}^{[4]}=\frac{x^{*}}{\delta-g} e^{\left(\delta-g-x^{*}\right) T} C_{T}^{[1]}$ \\
\hline$C_{T}(+)^{(a)}$ & $i d e m$ & $C_{T}^{[2]}=\frac{\Gamma^{\sigma} C_{T}^{[1]}}{1+e^{(g-\delta) T}\left(\Gamma^{\sigma}-1\right)}$ & $C_{T}^{[2]}=e^{\left(\delta-g-x^{*}\right) T} C_{T}^{[1]}$ \\
\hline$g(-)$ & $g=\sigma(\delta-\rho)$ & $g$ & $g^{[4]}=\delta-x^{*} \gtreqless g$ ssi $\Gamma \gtreqless 1$ \\
\hline$g(+)$ & $i d e m$ & $g$ & $g$ \\
\hline$\frac{C_{T}(+)}{C_{T}(-)}$ & 1 & $\Gamma^{\sigma} \gtreqless 1 \mathrm{ssi} \Gamma \gtreqless 1$ & $\frac{\delta-g}{x^{*}} \gtreqless 1 \mathrm{ssi} \Gamma \gtreqless 1$ \\
\hline$\frac{C_{t}}{R_{t}}(-)$ & $\delta-g$ & $\frac{\delta-g}{1+\left(\Gamma^{\sigma}-1\right) e^{(\delta-g)(t-T)}}$ & $x^{*}$ \\
\hline$\frac{C_{t}}{R_{t}}(+)$ & $i d e m$ & $\delta-g$ & $\delta-g$ \\
\hline
\end{tabular}

(a) on notera $X(-)$ et $X(+)$, les valeurs de la variable $X$ respectivement avant et après le changement en $T$.

(b) le calcul des valeurs des consommations stochastiques initiales et à la date de changement de préférences, obtenues par la résolution des problèmes $P[2]$ et $P[3]$, en fonction des consommations de référence correspondantes, solutions du problème $P[1]$, est présenté à l'annexe 5 .

(c) on suppose ici que l'incertitude est résolue en $T$.

Tout d'abord, lorsque $\Gamma=1$, c'est-à-dire lorsque le planificateur anticipe qu'en moyenneI les générations futures conserveront la même sensibilité à la consommation de ressource environnementale que les générations présentes, les trajectoires de consommation stochastique et déterministe sont clairement confondues et l'incertitude sur les préférences n'a aucune influence.

Lorsque $\Gamma>1$ en revanche, c'est-à-dire lorsque le planificateur anticipe que les générations futures auront en moyenne une préférence pour l'environnement plus grande que celle des générations présentes, le tableau 1 nous montre qu'il va s'écarter de la trajectoire de référence.

\section{(i) Date de changement des préférences certaine}

L'incertitude n'a pas d'effet sur le taux de croissance de la consommation avant le changement de préférences, mais seulement un effet de niveau. D'après les résultats résu- 
més dans le tableau 1 et représentés sur la figure 1, le niveau de consommation initial et celui qui prévaut juste avant le changement de préférences sont plus faibles sur la trajectoire stochastique [3] que sur la trajectoire de référence $\left(C_{0}^{[3]}<C_{0}^{[1]}\right.$ et $\left.C_{T}^{[3]}<C_{T}^{[1]}\right)$. Nous considérons que le planificateur est plus conservateur sur la trajectoire stochastique que sur la trajectoire de référence si le rapport de la consommation au stock de ressource est, à chaque instant, plus faible le long de la trajectoire stochastique. L'examen de ce rapport nous montre que si le planificateur anticipe une hausse de la sensibilité à l'environnement il adopte une attitude plus conservatrice avant la date de changement des préférences.

Le tableau 1 nous indique par ailleurs que $C_{T}^{[3]}<C_{T}^{[2]}$ : le niveau de consommation saute vers le haut au moment du changement de préférences. On peut aussi montrer que $C_{T}^{[2]}>C_{T}^{[1]}$ : le saut du niveau de consommation fait passer ce dernier au-dessus du niveau de consommation de référence, ceci pour compenser la moindre consommation initiale et assurer le respect de la contrainte de ressource intertemporelle. La consommation et le stock de ressource évoluent ensuite au taux déterministe $g$ (trajectoire [2]).

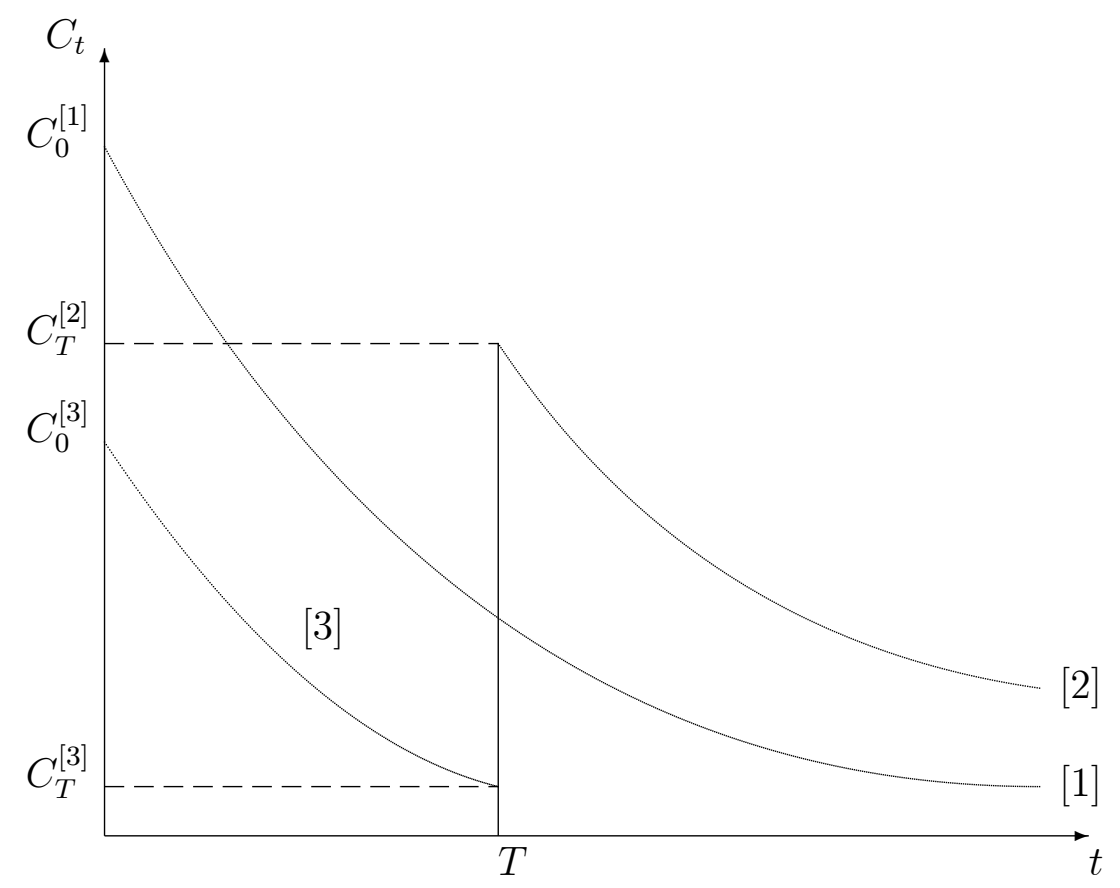

FIG. 1 - Trajectoires de consommation dans le cas $\Gamma>1$ quand la date de changement des préférences est certaine. Cas $g<0$

(ii) Cas où coexistent les deux incertitudes

Cette fois, l'incertitude a à la fois un effet de taux et un effet de niveau sur la consommation antérieure au changement de préférences.

La comparaison des consommations initiales est immédiate : $C_{0}^{[4]}<C_{0}^{[1]}$. De nouveau, le planificateur qui anticipe une hausse de la sensibilité des générations futures à l'environnement relativement à celle des générations présentes va initialement préserver davantage la ressource. En dépit du fait qu'au moment du changement de préférences il est possible que la consommation stochastique soit devenue plus élevée que la consommation détermi- 
niste $^{6}$, le planificateur sera toujours plus conservateur avant le changement de préférences, comme l'indique la valeur du ratio de la consommation au stock de ressource.

L'introduction d'une incertitude asymétrique sur les préférences des générations futures a aussi pour conséquence, quand $\Gamma>1$, d'augmenter le taux de croissance de la consommation de la période antérieure au changement de préférences, relativement au cas de référence. Il est même possible que les taux de croissance de la consommation avant et après le changement de préférences soient de signe opposé. C'est cette trajectoire nonmonotone de la consommation qui est représentée sur la figure 2 : après le changement de préférences, le taux de croissance est $g<0$ tandis qu'avant il vaut $g^{[4]}>0$. On voit aisément que ce cas est d'autant plus susceptible d'apparaître que $\Gamma$ est élevé.

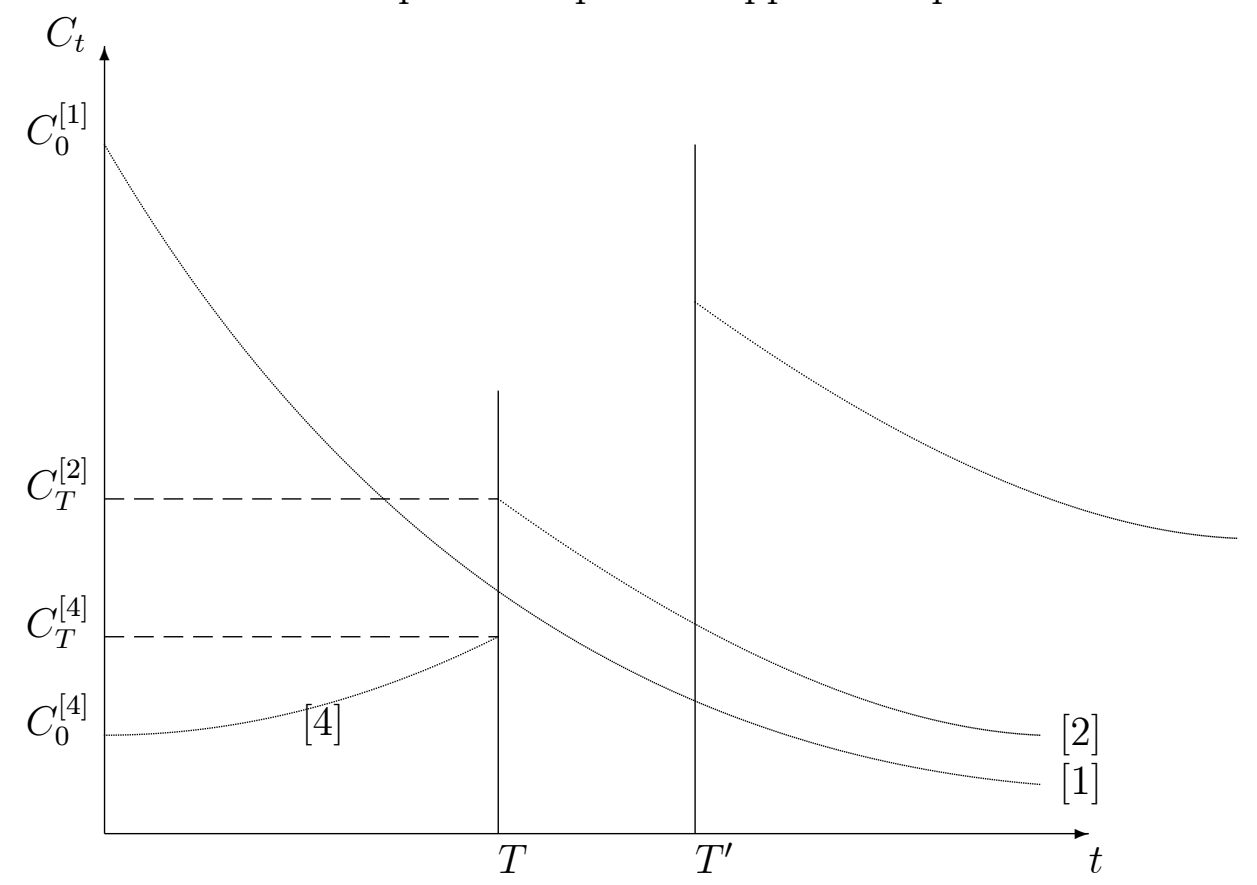

FIG. 2 - Trajectoires de consommation dans le cas $\Gamma>1$ quand la date de changement des préférences est incertaine

Pour achever la comparaison des trajectoires de référence [1] et stochastique [4] il faut enfin déterminer le sens et l'ampleur du saut de la consommation au moment du changement de préférences. A la date du changement de préférences ${ }^{7}$, le niveau de consommation va sauter pour que la contrainte intertemporelle de ressource soit satisfaite. Si l'on suppose que l'incertitude est résolue en $T$, le saut de la consommation au moment du changement de préférences est donné par :

$$
C_{T}^{[4]}-C_{T}^{[2]}=\left(g-g^{[4]}\right) R_{T}=\left(g-g^{[4]}\right) R_{0} e^{g^{[4]} T}<0
$$

Ainsi, quand $\Gamma>1$, ce saut se produit toujours vers le haut. Remarquons par ailleurs que l'ampleur du saut dépend évidemment de la date à laquelle advient le changement

\footnotetext{
${ }^{6}$ Ceci se produit si $C_{T}^{[4]}>C_{T}^{[1]} \Leftrightarrow \frac{x^{*}}{\delta-g}>e^{-\left(\delta-g-x^{*}\right) T} \Leftrightarrow T>\frac{1}{\delta-g-x^{*}} \ln \frac{\delta-g}{x^{*}}$ c'est-à-dire si la date à laquelle l'incertitude est résolue est suffisamment lointaine.

${ }^{7}$ Ce changement va survenir de façon certaine car nous avons supposé $\int_{0}^{\infty} f_{t} d t=1$.
} 
de préférences. On voit aisément que si le taux de croissance de la trajectoire antérieure au changement de préférence est positif, le saut est d'autant plus grand que la date du changement de préférences est éloignée de la date initiale. C'est ce cas qui est représenté sur la figure 2 (pour $T^{\prime}>T$ ). En revanche, si $g^{[4]}$ est négatif, le saut est d'autant plus petit que la date de changement de préférences est éloignée.

Le cas $\Gamma<1$ enfin est le symétrique du cas précédent : l'économie consomme initialement davantage, puis le niveau de consommation saute vers le bas au moment du changement de préférences; le planificateur est toujours moins conservateur qu'en l'absence d'incertitude.

\section{Conclusion}

Cet article étudie, dans un cadre très simple, les conséquences en termes de trajectoires optimales de consommation d'une incertitude sur les préférences des générations futures en matière d'exploitation d'une ressource environnementale renouvelable. Une telle incertitude va-t-elle induire un comportement optimal plus "conservateur" vis-à-vis de la ressource? Nous montrons que tel est le cas uniquement si nous anticipons aujourd'hui que les générations futures seront en moyenne plus sensibles que nous ne le sommes à la consommation permise par l'utilisation de la ressource environnementale. Nous montrons en outre que la prise en compte de cette incertitude conduit à une profonde modification de la trajectoire optimale de consommation, qui non seulement devient non-continue mais en outre peut devenir non-monotone.

\section{Références}

Ayong Le Kama, A. (2001) : "Preservation and Exogenous Uncertain Future Preferences," Economic Theory, 18(3), 745-752.

Ayong Le Kama, A., and K. Schubert (2004) : "Growth, Environment and Uncertain Future Preferences," Environmental and Resource Economics, 28(1), 31-53.

Beltratti, A. (1996) : Models of Economic Growth with Environmental Assets. Kluwer Academic Publishers, Fondazione ENI ENRICO MATTEI (FEEM) series on Economics, Energy and Environment.

Beltratti, A., G. Chichilnisky, and G. Heal (1993) : "Preservation, Uncertain Future Preferences and Irreversibility," Nota di Lavoro 59.93, Fondazione ENI ENRICO MATTEI (FEEM), Milan.

- (1998) : "Uncertain Future Preferences and Conservation," in Sustainability : Dynamics and Uncertainty, ed. by G. Chichilnisky, G. Heal, and A. Vercelli. Kluwer Academic Publishers, Fondazione Eni Enrico Mattei (FEEM) series on Economics, Energy and Environment. 
Dasgupta, P., and G. Heal (1974) : "The Optimal Depletion of Exhaustible Resources," Review of Economic Studies, pp. 3-28, Symposium Issue.

HeAL, G. (1985) : "Depletion and discounting : a classical issue in the economics of exhaustible resources," American Mathematical Sosiety, Pro Symposia App Math, $32,33-43$.

\section{Annexes}

\section{Annexe 1 : calcul de la valeur marginale de la ressource en $T$}

Si on reporte la valeur de la consommation optimale du problème $\mathcal{P}[2], C_{t}^{[2]}$, donnée par l'équation (13), dans l'expression de la fonction valeur, l'on obtient :

$$
\begin{aligned}
V\left(R_{T}\right) & =\int_{T}^{\infty} U\left[(\delta-g) R_{T} e^{g(t-T)}\right] e^{-\rho(t-T)} d t=U\left[(\delta-g) R_{T}\right] \int_{T}^{\infty} e^{\left(1-\frac{1}{\sigma}\right) g(t-T)} e^{-\rho(t-T)} d t \\
& =U\left[(\sigma \rho+(1-\sigma) \delta) R_{T}\right] \int_{T}^{\infty} e^{-(\sigma \rho+(1-\sigma) \delta)(t-T)} d t=\frac{U\left[(\sigma \rho+(1-\sigma) \delta) R_{T}\right]}{\sigma \rho+(1-\sigma) \delta}
\end{aligned}
$$

On a donc de façon évidente :

$$
V^{\prime}\left(R_{T}\right)=U^{\prime}\left(C_{T}^{[2]}\right)=\lambda_{T}^{[2]}
$$

\section{Annexe 2 : détermination des trajectoires optimales de consom- mation du modèle avec date de changement certaine}

Une façon alternative d'écrire l'équation d'évolution du stock de ressource environnementale (1) est la contrainte de ressource intertemporelle suivante :

$$
R_{0}=\int_{0}^{\infty} e^{-\delta t} C_{t} d t
$$

Cette contrainte peut s'écrire, entre 0 et une date $T$ quelconque,

$$
R_{0}=\int_{0}^{T} e^{-\delta t} C_{t} d t+e^{-\delta T} R_{T}
$$

Sur la trajectoire [3] antérieure au changement de préférences, la consommation croît au taux $g$, d'où la réécriture de la contrainte précédente :

$$
R_{0}=C_{0}^{[3]} \int_{0}^{T} e^{-(\delta-g) t} d t+e^{-\delta T} R_{T}=\frac{C_{0}^{[3]}}{\delta-g}\left[e^{-(\delta-g) T}-1\right]+e^{-\delta T} R_{T}
$$


dont on déduit :

$$
(\delta-g)\left(R_{T}-e^{\delta T} R_{0}\right)=C_{0}^{[3]}\left(e^{g T}-e^{\delta T}\right)
$$

En se rappelant que $C_{T}^{[3]}=C_{0}^{[3]} e^{g T}$ et $C_{T}^{[2]}=(\delta-g) R_{T}$ et en utilisant l'équation (19) du texte, on obtient la valeur suivante de la consommation initiale pour la trajectoire [3] :

$$
C_{0}^{[3]}=C_{T}^{[3]} e^{-g T}=\Gamma^{-\sigma} C_{T}^{[2]} e^{-g T}=\Gamma^{-\sigma}(\delta-g) R_{T} e^{-g T}
$$

Si on substitue cette expression de $C_{0}^{[3]}$ dans l'équation (A1), on obtient la valeur optimale du stock de ressource à la date du changement de préférences :

$$
R_{T}=\frac{R_{0}}{\Gamma^{-\sigma} e^{-g T}+e^{-\delta T}\left(1-\Gamma^{-\sigma}\right)}
$$

Cherchons à présent l'allure du sentier suivi par le stock de ressource avant le changement de préférences, entre $R_{0}$ et $R_{t}$. L'équation d'évolution de ce stock s'écrit, en utilisant l'équation (20) :

$$
\dot{R}_{t}=\delta R_{t}-C_{t}^{[3]}=\delta R_{t}-(\delta-g) e^{g(t-T)} \Gamma^{-\sigma} R_{T}
$$

Ceci s'intègre aisément en :

$$
R_{t}=\left(R_{0}-e^{-g T} \Gamma^{-\sigma} R_{T}\right) e^{\delta t}+\Gamma^{-\sigma} R_{T} e^{g(t-T)}
$$

que l'on peut réécrire en utilisant l'équation (A2) sous la forme de l'équation (22) du texte.

\section{Annexe 3 : détermination du profil de consommation optimale du problème $\mathcal{P}[4]$}

On écrit le hamiltonien courant du problème $\mathcal{P}[4]$ (en omettant l'indice temporel) :

$$
\mathcal{H}=[1-F] U(C)+f \Gamma V(R)+\lambda(\delta R-C)
$$

Les conditions nécessaires d'optimalité sont :

$$
\left\{\begin{array}{l}
\lambda=[1-F] U^{\prime}(C) \\
\dot{\lambda}=(\rho-\delta) \lambda-f \Gamma V^{\prime} \\
\dot{R}=\delta R_{t}-C \\
\lim _{t \rightarrow \infty} e^{-\rho t} \lambda_{t} R_{t}=0
\end{array}\right.
$$

La différentiation de l'équation $(i)$ donne :

$$
\frac{\dot{\lambda}}{\lambda}=-\frac{f}{1-F}+\frac{C U^{\prime}(C)}{U^{\prime}(C)} \frac{\dot{C}}{C} \Longleftrightarrow-\frac{1}{\sigma} \frac{\dot{C}}{C}=\frac{\dot{\lambda}}{\lambda}+\frac{f}{1-F}
$$

d'où, en utilisant $(i i)$ et $(i)$ :

$$
-\frac{1}{\sigma} \frac{\dot{C}}{C}=(\rho-\delta)-\frac{f}{\lambda} \Gamma V^{\prime}+\frac{f}{1-F}=\rho-\delta+\frac{f}{1-F}\left[1-\frac{\Gamma V^{\prime}}{U^{\prime}(C)}\right]
$$


Si l'on note $\mu_{t}=\frac{f_{t}}{1-F_{t}}$ la probabilité conditionnelle que le changement se réalise en $t$ sachant qu'il s'est pas fait avant, on a ainsi :

$$
\frac{\dot{C}_{t}^{[4]}}{C_{t}^{[4]}}=g-\sigma \mu_{t}\left[1-\frac{\Gamma V^{\prime}}{U^{\prime}\left(C_{t}^{(4)}\right)}\right]
$$

\section{Annexe 4 : l'état stationnaire du problème $\mathcal{P}[4]$}

Reprenons l'équation (30). Si l'on note $a=g-\sigma \mu-\delta<0$ (car $g-\delta<0$ par hypothèse) et $b=\sigma \mu \Gamma(\delta-g)^{-\frac{1}{\sigma}}>0$, elle s'écrit :

$$
\frac{\dot{x}_{t}}{x_{t}}=a+b x_{t}^{\frac{1}{\sigma}}+x_{t}
$$

Nous cherchons l'état stationnaire associé à cette équation. Soit $h(x)=a+b x^{\frac{1}{\sigma}}+x$. On a $h^{\prime}(x)>0, h(0)=a<0, h(\infty)=+\infty$, et $h^{\prime \prime}(x)>0$ si $\sigma<1$ et $<0$ dans le cas contraire. La fonction $h$ est donc monotone et croissante, et prend ses valeurs entre $a=-[(1-\sigma) \delta+\sigma(\rho+\mu)]<0$ et $+\infty$. Ainsi, il existe une valeur unique notée $x^{*}$ telle que $h\left(x^{*}\right)=0$ : l'équation (30) admet un état stationnaire unique (voir la figure 3).

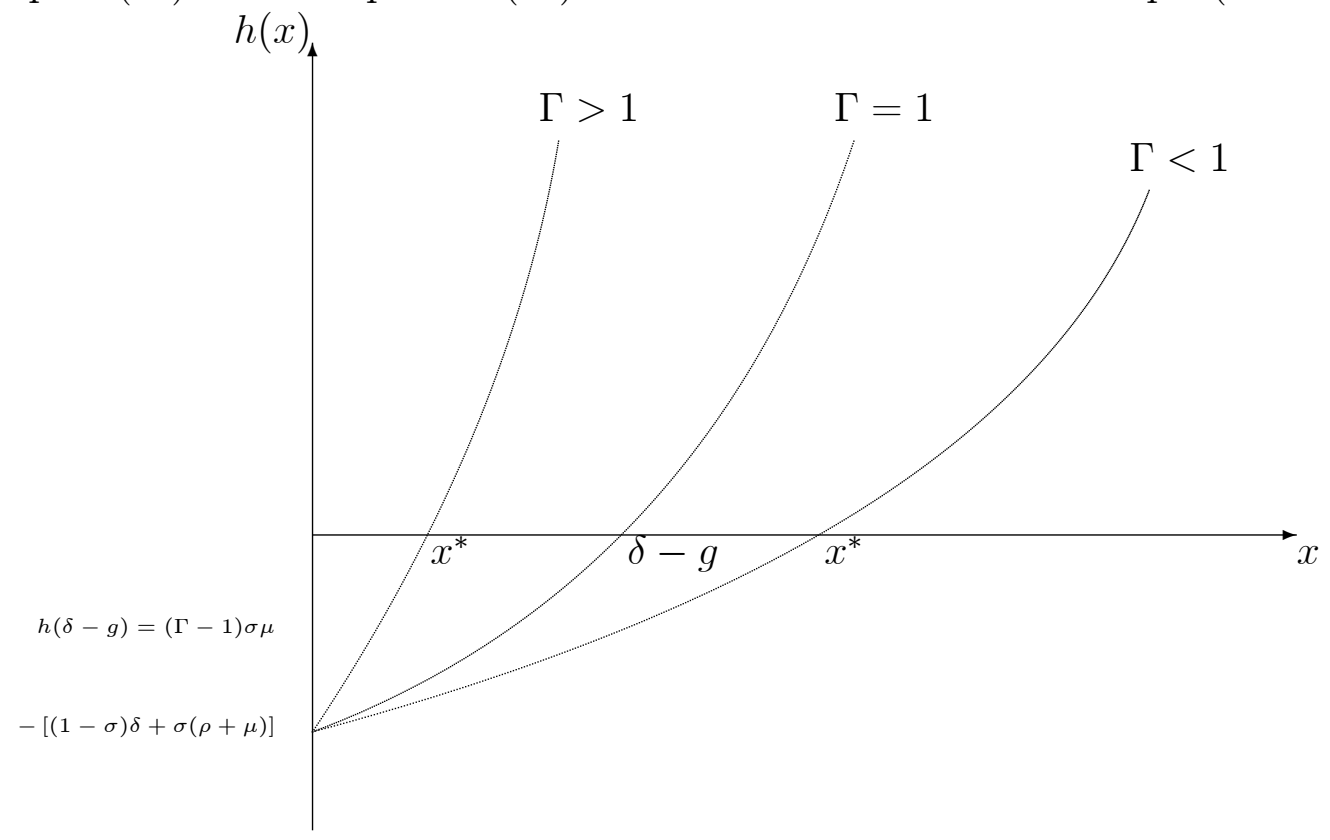

FIG. 3 - Solution stationnaire de l'équation différentielle en $x$; cas $\sigma<1$

Notons que dans le cas sans changement de préférences (ou, de façon équivalente, quand $\Gamma=1)$ cet état stationnaire est $\delta-g$. Calculons alors $h(\delta-g)$ :

$h(\delta-g)=a+b(\delta-g)^{\frac{1}{\sigma}}+(\delta-g)=g-\sigma \mu-\delta+\sigma \mu \Gamma(\delta-g)^{-\frac{1}{\sigma}}(\delta-g)^{\frac{1}{\sigma}}+(\delta-g)=(\Gamma-1) \sigma \mu$

Donc :

$$
h(\delta-g)\left\{\begin{array} { l } 
{ = 0 \text { si } \Gamma = 1 } \\
{ > 0 \text { si } \Gamma > 1 } \\
{ < 0 \text { si } \Gamma < 1 }
\end{array} \quad \text { d'où } x ^ { * } \left\{\begin{array}{l}
=\delta-g \text { si } \Gamma=1 \\
<\delta-g \text { si } \Gamma>1 \\
>\delta-g \text { si } \Gamma<1
\end{array}\right.\right.
$$


Enfin, l'équation (30) est instable : le ratio $C / R$ se place immédiatement à sa valeur stationnaire $x^{*}$. On a donc :

$$
g^{[4]}=\frac{\dot{C}^{[4]}}{C^{[4]}}=\delta-x^{*}\left\{\begin{array}{l}
=g \text { si } \Gamma=1 \\
>g \text { si } \Gamma>1 \\
<g \text { si } \Gamma<1
\end{array}\right.
$$

\section{Annexe 5 : comparaison des trajectoires de consommation issues}

\section{des problèmes $\mathcal{P}[1]$ et $\mathcal{P}[3]$}

On détermine tout d'abord la consommation initiale stochastique, $C_{0}^{[3]}$, en fonction de la consommation initiale déterministe, $C_{0}^{[1]}$. On sait que $C_{0}^{[3]}=\Gamma^{-\sigma}(\delta-g) R_{T} e^{-g T}$ et, en substituant le stock de ressource à la date de changement par sa valeur donnée en (A2) on obtient :

$$
C_{0}^{[3]}=\frac{(\delta-g) R_{0} \Gamma^{-\sigma} e^{-g T}}{\Gamma^{-\sigma} e^{-g T}+e^{-\delta T}\left(1-\Gamma^{-\sigma}\right)}
$$

Sachant que $C_{0}^{[1]}=(\delta-g) R_{0}$, cette équation devient :

$$
C_{0}^{[3]}=\frac{\Gamma^{-\sigma} e^{-g T}}{\Gamma^{-\sigma} e^{-g T}+e^{-\delta T}\left(1-\Gamma^{-\sigma}\right)} C_{0}^{[1]}=\frac{1}{1+e^{(g-\delta) T}\left(\Gamma^{\sigma}-1\right)} C_{0}^{[1]}
$$

On détermine ensuite les niveaux de consommation pour les trajectoires [3] et [2], $C_{T}^{[3]}$ et $C_{T}^{[2]}$, au moment du changement de préférences, en fonction du niveau de consommation déterministe à la même date, $C_{T}^{[1]}$. On sait que $C_{T}^{[1]}=(\delta-g) R_{0} e^{g T}$. On sait par ailleurs que :

$$
C_{T}^{[2]}=(\delta-g) R_{T}=\frac{(\delta-g) R_{0}}{\Gamma^{-\sigma} e^{-g T}+e^{-\delta T}\left(1-\Gamma^{-\sigma}\right)}=\frac{1}{1+e^{(g-\delta) T}\left(\Gamma^{\sigma}-1\right)} \Gamma^{\sigma} C_{T}^{[1]}
$$

et, par déduction, on obtient :

$$
C_{T}^{[3]}=\frac{1}{1+e^{(g-\delta) T}\left(\Gamma^{\sigma}-1\right)} C_{T}^{[1]}
$$

